\title{
Epidermolysis bullosa Associated with Type 1 Diabetes Mellitus - Case Report of a Lethal Disease
}

\author{
Claudia Jurca',2*, Marius Bembea ${ }^{1}$, Kinga Kozma ${ }^{1,2}$, Alfred Klausegger ${ }^{3}$, Ariana Szilagyi ${ }^{1,2}$ and \\ Alexandru Jurca ${ }^{2}$
}

${ }^{1}$ Municipal Clinical Hospital “Dr. Gavril Curteanu” Oradea, Romania

${ }^{2}$ Department of Preclinical Specialties, Faculty of Medicine and Pharmacy, University of Oradea, Romania

${ }^{3}$ EB House Austria, Laboratory of Molecular Therapy, University Hospital of the Paracelsus Medical University, 5020 Salzburg, Austria

*Corresponding author: Claudia Jurca, Department of Preclinical Specialties, Faculty of Medicine and Pharmacy, University of Oradea, 1 Decembrie Square, 410068 Oradea, Bihor County, Romania, Tel: +0040744671306; Fax: +40259442687; E-mail: claudiajurca70@yahoo.com

Received date: March 02, 2018; Accepted date: March 15, 2018; Published date: March 22, 2018

Citation: Jurca C, Bembea M, Kozma K, Szilagyi A, Jurca A, et al. (2018) Epidermolysis bullosa Associated with Type 1 Diabetes Mellitus - Case Report of a Lethal Disease. J Rare Disord Diagn Ther. Vol. 4 No. 1: 6.

Copyright: @ 2018 Jurca C, et al. This is an open-access article distributed under the terms of the Creative Commons Attribution License, which permits unrestricted use, distribution, and reproduction in any medium, provided the original author and source are credited.

\begin{abstract}
We present a rare lethal case of a dystrophic form of epidermolysis bullosa (EB) associated with type 1 diabetes mellitus in a 5-year-old boy diagnosed with EB at birth. This association has not been reported before. Two wellknown mutations in the collagen COL7A1 gene (compound heterozygous: c.425A $>\mathrm{G}$ in exon 3 and $\mathrm{c}$. $2005 C>T$ in exon 15) were determined as cause of EB. Whether the association with diabetes mellitus type 1was causally related remained undetermined. We also discuss the therapeutic difficulties resulting from this association.
\end{abstract}

Keywords: Epidermolysis bullosa; Diabetes mellitus; COL7A1 gene

\section{Introduction}

Epidermolysis bullosa (EB) is a heterogeneous group of diseases characterized by blisters of the skin and mucosal membranes ranging from mild to lethal [1]. It results from an anchoring defect between the epidermis and dermis, resulting in friction and skin fragility [2] About 30 genetically different types share, as a common feature, this mechanical fragility of epithelial tissues, most notably the skin [3]. Based on electron microscopy, EB is classified into three categories according to the level where blisters are formed: simplex EB - blisters formed on the basal layer; junctional EB - blisters formed in the lamina lucida; dystrophic EB (DEB) - blisters formed in dense lamina [4]. Aside from various degrees of severity of blistering other complications can also occur, including pain, anemia, esophageal stenosis or cardiomyopathy. Patients with missing collagen protein are at risk of cutaneous malignancies at adult age. The majority of patients with dystrophic EB will develop cutaneous squamous carcinoma by the age of 40 years [5].

Genetically, two forms of DEB have been described: an autosomal recessive form (OMIM 226600) and an autosomal dominant form (OMIM 131750).

Type 1 diabetes mellitus (DM1) is a polygenic multifactorial disorder resulting from autoimmune destruction of pancreatic beta cells producing insulin. Multiple genes and environmental factors such as viruses, some dietary factors, certain chemicals or drugs have been implied in its etiology [6]. The association of these EB and type 1 diabetes mellitus has very rarely been described in the literature [7].

\section{Patient and Methods}

The patient was referred to our genetics service on the 7th day of life in May 2009 by, family doctor, primary care physician. The diagnosis of EB was based on the characteristic clinical signs present at birth: bullous blistering formation occurring immediately after birth, in areas exposed to mechanical and even minor trauma. Electronic microscopy examination failed to yield results. Peripheral blood (EDTA) for genetic testing was obtained from the patient as well as his parents after informed consent. DNA was extracted using a DNA isolation kit according to the manufacturer protocol (Omega, Georgia, USA). Mutation detection and screening of exons 1-48 and exons 72-118 of the COL7A1 gene of the index patient was performed using the "priority strategy." The coding region and adjacent intron/exon boundaries specific for the COL7A1 gene of the patient were amplified by PCR, sequenced by Sanger and compared to the database (reference for exons and mRNA: NM000094; CDS 2-8836). At the age of 16 months the patient was suspected to have developed type 1 diabetes mellitus based on suggestive clinical signs: thirst, weight loss, polyphagia, polyuria and frequent urination. The diagnosis was confirmed by biochemical tests: increased blood glucose levels 
of $134-615 \mathrm{mg} / \mathrm{dl}$ (reference values $60-99 \mathrm{mg} / \mathrm{dl}$ ), glycosuria $(2+)$, ketonuria $(2+)$, metabolic acidosis pH 7.22 (ref. $\mathrm{pH}$ 7.35-7.45), glycated hemoglobin (14\%); islet cells antibodies and insulin antibodies were present.

\section{Results}

The patient, born on May 2009, presented from the first days of life with congenital multiple cutaneous forms of blisters on the limbs, trunk, face and on the buccal mucosa. The blisters size differed from $1-2 \mathrm{~cm}$ to $10 \mathrm{~cm}$ diameter. They broke open and involved broad epidermal areas of erosion, ulceration, and purulent secretions.

The patient was the first child in a family of unrelated healthy parents. There are no other known cases of illness or other significant diseases in the family. The pregnancy had a normal course. Delivery was normal without signs of hypoxia. During the course of the disease multiple new blisters appeared over the entire skin, seemingly spontaneous or caused by minor trauma. This caused multiple wounds and scars deforming areas of skin. Subsequently atrophy of the nails and multiple scars developed some retractile, with a tendency of synechiae and abnormal semi-flexion position of the fingers. The lesions were intensely painful and pruritic. Blistering also involved the eyes and mucosa of the mouth. The patient manifested retardation of the gross motor skills because of pain and blistering. Tentative skin biopsy and electron microscopy examination failed technically.

At the age of 16 months, he was diagnosed with diabetes mellitus type 1 requiring insulin substitution using an insulin pump initiated with an adequate dietary regime. Patient compliance to treatment was appropriate, diabetes remains under control in clinical and biological balance.

Molecular analysis identified a compound mutation in the COL7A1 gene (Collagen VII, alpha-1 polypeptide): c.425A>G, p.K142R, exon 3 of maternal origin and c.2005C>T, p.R669X, exon 15 of paternal origin (Figure 1).

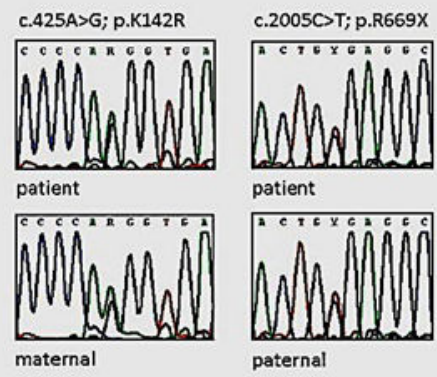

Figure 1: Molecular analysis revealed the same mutation c. $425 A>G$ in the mother and son (described first time by Gardella in 1996) and the mutation c.2005C >T in the father and son (described first time by Cserhalmi-Friedman in 1998).

\section{Discussion}

Our observation refers to two different genetic entities not known to be causally related. The dystrophic form of EB described here is an autosomal recessive disease caused by mutations in the gene COL7A1 located on chromosome 3p21.31 (Gene MIM number 120120). Christiano et al. described the entire intron/exon organization of COL7A1 in 1994 [8]. This gene has 118 exons, one of the genes with the highest number of exons [9]. In the DEB register (https:// www.deb-central.org/) 659 mutations are listed whereas in the Human Gene Mutation Database (HGMD) professional 2017 a total of 773 mutations are registered (http:// www.hgmd.cf.ac.uk/ac/gene.php?gene=COL7A1). These mutations alter the structure or disrupt the production of type VII collagen, which impairs the ability of anchoring fibrils to connect the epidermis to the dermis $[10,11]$. Of the two mutations in COL7A1 in our patient, c.425A>G in exon 3 has been described previously by Gardella et al. in 1996 [12]. This splice site mutation, $425 \mathrm{~A} \rightarrow \mathrm{G}$, has been observed repeatedly in Italian and Central European populations $[13,14]$. Varki et al. in 2007 analyzed COL7A1 gene mutations in 310 patients with the referral diagnosis of DEB on the basis of clinical observations, evidence for sublamina densa blistering, negative or attenuated immunofluorescence for type VII collagen or ultrastructural abnormalities in the anchoring fibrils. They found a mutation in one or both alleles in 243 (78.4\%) of patients. The mutation most frequently detected was c. $425 \mathrm{~A}>\mathrm{G}$ in $3.9 \%$ as occurred in our patient. As with other splice-site mutations in COL7A1 causing a premature codon termination, the phenotypic expression is similar to our patient, namely, a severe clinical type of EBD.

Mutation c. $2005 \mathrm{C}>\mathrm{T}$, exon 15 was described for the first time by Cserhalmi-Friedman et al. in 1998 [15]. This rare heterozygous variant is associated with different phenotypes: short stature, syndactyly of fingers and toes, and palmoplantar blistering. Our patient had no growth delay, no finger and toe syndactyly, and the blistering was generally distributed.

Our patient is compound heterozygous, each of the two parents contributing a different mutation in the same gene. The inheritance pattern corresponds to autosomal recessive inheritance. Dystrophic forms of EB, even if it is not proved by electron microscopy, can be established by the clinical aspects and the mutations identified.

Hirsch et al. described recently a considerable success in somatic gene therapy in a 5-year-old patient with junctional epidermolysis bullosa due to a homozygous mutation in the LAMB3 gene (OMIM 150310). Cultured keratinocytes from a 4 $\mathrm{cm} 2$ biopsy of unaffected skin were transfected with a retroviral vector carrying the normal $L A M B 3$ gene, grown to $0.85 \mathrm{~cm}^{2}$, grafted to the patient. The transgenic skin transplantation restored normal skin function [16].

Diabetes mellitus type 1 is a polygenic multifactorial disease involving multiple genes and environmental factors. The most important insulin-dependent (type I) diabetes mellitus (IDDM1 gene) is located on chromosome 6 p21 within MHC, class II 
region (histocompatibility system). Most DM1 susceptibility alleles are in MHC class II DR and DQ genes, located very close to each other, forming the DR-DQ haplotype. Increased risk for DM1 is attributed to the DR3-DQ2 and DR4-DQ8 haplotypes. The two haplotypes present together are found in $30-50 \%$ of patients with $\mathrm{DM} 1$ versus $2-3 \%$ in the general population [17]. Among the protective DR-DQ haplotypes, the DR7 haplotype bearing DQB1 is significantly more protective than the one bearing DQB1.

At the current level of knowledge of the human genome the combination of the two entities described her have to be considered as coincidental. Klausegger communicated 4 patients ( 3 patients from Bosnia and Herzegovina and 1 patient from Serbia) with the same mutation combination: $c$. $425 A>G, p . K 142 R$, exon 3 and c.2005C >T, p.R669X, exon 15 (personal communication). All 4 patients did not show any signs of associated DM1. However, a causal relationship of the two diseases cannot be excluded. There are few factors that could be involved in this case: e.g. different bacterial and fungal germs causing prolonged and repeated infections, the use of many and different antibiotics or diverse and massive use of antiseptic substances [18].

The association of the two disorders raises other complicated issues related to the use of insulin and the diet. The usage of the insulin pump, however, is abrogating all its drawbacks related to cost and accessibility. In addition, it is recommended to use especially soft foods avoiding causing oral-esophageal mucosal injury, which can create difficulties to establish this sick menu [19].

The clinical course in our patient was unfavorable, with multiple, generalized bullous lesions, scars, constrictions, major difficulties with food intake, need for gastrostomy. Gradual worsening of symptoms and persistent complications (severe malnutrition, hydroelectrolytic imbalance, hypoproteinemia, infections) led to death at the age of 5 years and 6 months.

\section{Conclusion}

An association of dystrophic epidermolysis bullosa and type 1 diabetes mellitus as described here is a rare event. It is most probably a non-causal coincidence. Dystrophic EB associated with diabetes mellitus type 1 requires a particular therapeutic concept ensuring both continuous administration of insulin and an optimal dietary program.

\section{Acknowledgment}

We thank Professor Eberhard Passarge, Essen, Germany, for support with the text and helpful suggestions.

\section{References}

1. Fine JD, Bruckner-Tuderman L, Eady RA, Bauer EA, Bauer JW, et al. (2014) Inherited epidermolysis bullosa: updated recommendations on diagnosis and classification. J Am Acad Dermatol 70: 1103-1126.
2. Varki R, Sadowski S, Uitto J, Pfendner E (2007) Epidermolysis bullosa. II. Type VII collagen mutations and phenotypegenotype correlations in the dystrophic subtypes. J Med Genet 44: 181-92.

3. Irvine AD (2005) Inherited defects in keratins. Clin Dermatol 23: 6-14.

4. Intong LR, Murrell DF (2012) Inherited epidermolysis bullosa: new diagnostic criteria and classification. Clin Dermatol 30: 70-7.

5. Mallipeddi R (2002) Epidermolysis bullosa and cancer. Clin Exp Dermatol 27: 616-23.

6. de Beeck AO, Eizirik DL (2016) Viral infections in type 1 diabetes mellitus--why the $\beta$ cells? Nat Rev Endocrinol 12: 263-73.

7. Park K, Nakano H, Sawamura D, Kabashima K, Miyachi Y, et al. (2014). A case of dominant dystrophic epidermolysis bullosa with diabetes mellitus presenting as progressive recalcitrant blisters and erosions. Int Wound J 11: 567-568.

8. Christiano AM, Hoffman GG, Chung-Honet LC, Lee S, Cheng W, et al. (1994) Structural organization of the human type VII collagen gene (COL7A1), composed of more exons than any previously characterized gene. Genomics 21: 169-79.

9. Lin Y, Xue-Jun C, Liu W, Gong B, Xie J, et al. (2012) Two Novel Mutations on Exon 8 and Intron 65 of COL7A1 Gene in Two Chinese Brothers Result in Recessive Dystrophic Epidermolysis Bullosa. PLo S One 7: e505.

10. Wertheim-Tysarowska K, Sobczynska-Tomaszewska A, Kowalewski C, Skronski M, Swieckowski G, et al. (2012) The COL7A1 mutation database. Hum Mutat 33: 327-331.

11. Christiano AM, Anton-Lamprecht I, Amano S, Ulrike E, Burgeson Robert E, et al. (1996) Compound heterozygosity for COL7A1 mutations in twins with dystrophic epidermolysis bullosa: a recessive paternal deletion/ insertion mutation and a dominant negative maternal glycine substitution result in a severe phenotype. Am J Hum Genet 58: 682-693.

12. Gardella R, Belletti L, Zoppi N, Marini D, Barlati S, et al. (1996) Identification of two splicing mutations in the collagen type VII gene (COL7A1) of a patient affected by the localisata variant of recessive dystrophic epidermolysis bullosa. Am J Hum Genet 59: 292-300.

13. Gardella R, Castiglia D, Posteraro P, Bernardini S, Zoppi N, et al. (2002) Genotype-phenotype correlation in Italian patients with dystrophic epidermolysis bullosa. J Invest Dermatol 119: 1456-62.

14. Csikós $M$, Szöcs HI, Lászik $A$, Mecklenbeck $S$, Horváth $A$, et al. (2005) High frequency of the 425 AR G splice-site mutation and novel mutations of the COL7A1 gene in central Europe: significance for future mutation detection strategies in dystrophic epidermolysis bullosa. Br J Dermatol 152: 879-886.

15. Cserhalmi-Friedman PB, McGrath JA, Mellerio JE, Romero R, Salas-Alanis JC, et al. (1998) Restoration of open reading frame resulting from skipping of an exon with an internal deletion in the COL7A1 gene. Lab Invest 78: 1483-92.

16. Hirsch T, Rothoeft T, Teig N, Bauer JW, Pellegrini G, et al. (2017) Regeneration of the entire human epidermis using transgenic stem cells. Nature 551: 327-332.

17. Covic M, Sandovici I, Ștefănescu D, Gorduza EV (2017) Genetica Medicală; capitolul 14; Editura Polirom lași pg. 448-449. 
18. Filippi Christophe M, von Herrath Matthias G (2008) Viral Trigger for Type 1 Diabetes. Diabetes 57: 2863-2871.

19. Parushetti AD, Agrawal JM, Nanjannawar LG, Agrawal MS (2013) Oral manifestations of epidermolysis bullosa dystrophica: a rare genetic disease. BMJ Case Rep 2013: bcr2012007963. 